\title{
Desarrollo de la competencia mediática de los estudiantes en el contexto de la educación digital
}

\section{Development of students' media competence in the context of digital education}

\author{
Marina R. Zheltukhina \\ zzmr@mail.ru \\ Volgograd State Socio-Pedagogical University, Volgograd, Russia \\ https://orcid.org/0000-0001-7680-4003 \\ Maxim M. Kutepov \\ kmm-asb@mail.ru \\ Minin Nizhny Novgorod State Pedagogical University, Nizhny Novgorod, Russian Federation \\ https://orcid.org/0000-0002-5397-6168 \\ Lyubov I. Kutepova \\ lubovkutepova@mail.ru \\ Minin Nizhny Novgorod State Pedagogical University, Nizhny Novgorod, Russian Federation \\ https://orcid.org/0000-0002-3175-4978 \\ Marina N. Bulaeva \\ bulaevamarina@mail.ru \\ Minin Nizhny Novgorod State Pedagogical University, Nizhny Novgorod, Russian Federation \\ https://orcid.org/0000-0002-9928-9451

\section{Anna V. Lapshova} \\ any19.10@mail.ru \\ Minin Nizhny Novgorod State Pedagogical University, Nizhny Novgorod, Russian Federation \\ https://orcid.org/0000-0001-7017-3589
}

Recibido: $10 / 12 / 2020$

Aceptado: 13/01/2021

\section{Resumen}

El presente artículo tuvo como objetivo el análisis de la experiencia de formación de la competencia mediática de los estudiantes en el contexto de la educación digital. La metodología involucró a estudiantes de educación superior buscando determinar la confiabilidad de los resultados de la dinámica de formación de la competencia mediática en los grupos de control y experimentales se llevó a cabo probando la hipótesis estadística de comparar la media mediante la prueba t de Student. Lo cual permite revelar las posibilidades de las tecnologías de la información en la preparación de los estudiantes modernos, para determinar el desarrollo de la competencia mediática en dinámica. 
Marina R. Zheltukhina, Maxim M. Kutepov, Lyubov I. Kutepova, Marina N. Bulaeva, Anna V. Lapshova.

Palabras clave: competencia mediática, educación mediática, tecnología de la información, educaci ón digital, tecnología electrónica, educación profesional.

\section{Abstract}

The objective of this article was to analyze the experience of training students' media competence in the context of digital education. The methodology involved higher education students seeking to determine the reliability of the results of the dynamics of media competence formation in the control and experimental groups, it was carried out by testing the statistical hypothesis of comparing the mean using the Student's t test. Which allows to reveal the possibilities of information technologies in the preparation of modern students, to determine the development of media competence in dynamics.

Key Words: media competence, media education, information technology, digital education, electronic technology, professional education.

\section{Introducción}

Los requisitos de los estándares educativos estatales federales modernos indican la necesidad del desarrollo y uso activo de tecnologías educativas electrónicas en la formación de estudiantes de instituciones de educación superior. Con la transición a la educación a distancia, es necesario utilizar medios técnicos adicionales para organizar el proceso educativo. Las plataformas de aprendizaje electrónico permiten a los estudiantes completar las tareas adjuntando los archivos correspondientes al curso electrónico. El profesor puede comprobarlos rápidamente y hacer los ajustes necesarios. El uso de aplicaciones de webinars posibilitó la realización de clases online en condiciones próximas a la formación presencial. Cada alumno se une a la conferencia a través de una invitación del profesor.

La creciente importancia de las tecnologías electrónicas y su amplia introducción en la vida de la sociedad determina la demanda práctica de competencia mediática como una herramienta que le permite llevar a cabo de manera efectiva varios tipos de actividades (Vaganova et al., 2020). El desarrollo de la competencia mediática de los estudiantes es una de las principales tareas que resuelven las instituciones educativas profesionales.

El aprendizaje a distancia implica el estudio modular de cursos por parte de los estudiantes, lo que le permite organizar el proceso de aprendizaje según las necesidades individuales y grupales de los estudiantes. La flexibilidad del aprendizaje a distancia se logra mediante el uso de soluciones tecnológicas innovadoras que los estudiantes necesitan utilizar tanto en el proceso de aprendizaje como en sus futuras actividades profesionales. Estas soluciones permiten que los sujetos del proceso educativo interactúen libremente, realicen tareas asignadas, participen en concursos profesionales, conferencias y realicen proyectos grupales e individuales. 
El desarrollo de la competencia mediática satisfará la necesidad del mercado laboral de especialistas preparados para el uso profesional de las tecnologías de la información, que se orienten rápidamente en el espacio global de la información (Shcerbakova et al., 2019).

La competencia mediática implica la motivación de los estudiantes, el interés en el uso de tecnologías electrónicas, el conocimiento de los medios por parte de los estudiantes, la posesión de habilidades analíticas y reflexivas, la capacidad para evaluar la argumentación y la selección de información relevante. Un factor importante en la formación de la competencia mediática es la observancia de las relaciones asignaturaasignatura de los alumnos con los profesores, que activan la independencia de los alumnos, su posición creativa y su implicación en el proceso educativo.

Para el desarrollo de la competencia mediática, es necesario prestar atención a la organización y equipamiento del entorno educativo multimedia de la Universidad, su equipamiento. Los estudiantes no solo deben navegar por el espacio de Internet, seleccionar y estructurar información relevante, sino también participar en proyectos de medios (investigación, creatividad, juegos, educativos y cognitivos, etc.). Los proyectos de medios en el entorno educativo moderno son productos de medios independientes hechos bajo la supervisión de un maestro que utiliza tecnologías de la información innovadoras.

En el contexto del desarrollo del aprendizaje digital, han aparecido muchas herramientas electrónicas que se pueden utilizar en el proceso educativo, incluso cuando los estudiantes crean proyectos de medios (Yarygin et al., 2019b). Se trata de plataformas para el aprendizaje a distancia, webinars, programas para la creación de cuadros y gráficos, mensajeros para el intercambio rápido de información.

Internet proporciona un acceso rápido a varias bases de datos, pero la información propuesta a menudo no está actualizada, una de las razones es la alta tasa de actualización. La competencia mediática resuelve el problema de seleccionar y utilizar información relevante por parte del alumno. La competencia mediática es parte de la competencia profesional de un especialista competitivo moderno.

\section{Marco teórico}

A pesar de una amplia gama de investigadores que han dedicado su trabajo al estudio de la competencia mediática, el tema de su formación y desarrollo en los estudiantes de educación superior sigue estando insuficientemente desarrollado. Con la transición al proceso de aprendizaje a distancia, los estudiantes de diferentes cursos se vieron obligados a adaptarse rápidamente a las tecnologías electrónicas y utilizarlas en el proceso de formación (Pichugina et al., 2019). 
El término "medi os" se utiliza con mayor frecuencia como sinónimo de medios de comunicación masiva, que incluyen fotos, videos, sistemas informáticos multimedia e Internet, entre otros.

La multimedia se define en diferentes valores: como un medio de procesamiento y almacenamiento de información, como un conjunto de medios técnicos, cuya finalidad es reproducir simultáneamente varios tipos de información, como un producto de información final. En todos los casos presentados, la multimedia es una parte integral de la formación de la competencia mediática del estudiante (Nagovitsyn et al., 2020).

La multimedia combina varias formas de percibir la información (sonido, texto, sensaciones táctiles al interactuar con entornos interactivos) (Yarygin et al., 2019a). Existen los siguientes tipos de multimedia: software, equipos multimedia, entornos de formación.

Las tecnologías multimedia son un conjunto de tecnologías de la información y la comunicación que permiten el uso de todo tipo de información, como textos, audio y video, gráficos, dibujos, animación y tablas (Korostelev et al., 2019).

Muchos autores hablan de multimedia como un conjunto de herramientas de software y hardware que permiten procesar información en una forma fácil de entender: visual y de audio (Bogdanova et al., 2019), (Vaganova et al., 2019).

SV Petrova presenta la competencia mediática como un sistema dinámico de valores, un conjunto de cualidades personales, experiencia, conocimientos, habilidades y habilidades de los sujetos del proceso educativo para comprender y crear textos mediáticos educativos y artísticos en un entorno educativo multimedia (Petrova, 2018)

La competencia mediática también se considera la capacidad de utilizar, analizar y evaluar información en diversas formas. Los medios en su conjunto se revelan como un medio y como un recurso y como un medio (Dobudko et al., 2019b). La competencia mediática implica la formación de una cultura de comunicación con los medios, habilidades comunicativas, interpretación y análisis de textos mediáticos (Kidina, 2020).

La importancia de la educación en medios se enfatiza en sus trabajos de S. G. Grigoriev (Grigoriev, 2005), N. V. Zmanovskaya (Zmanovskaya, 2004), N. A. Konovalova (Konovalova, 2004) y otros. La formación de la competencia mediática se considera en el marco del desarrollo de la alfabetización mediática y las habilidades mediáticas (Rojas-Bahamón et al., 2019). Los autores señalan que la competencia en medios le permite utilizar de manera competente las tecnologías de la información y no solo obtener acceso a diversas fuentes de información, sino también crear sus productos de medios que se pueden utilizar en futuras actividades profesionales. 
N. V. Zmanovskaya considera que la competencia mediática es un conjunto de conocimientos, habilidades y formación de ideas sobre los medios, comprensión de la esencia de la competencia mediática (Zmanovskaya, 2004).

La formación de la competencia mediática está asociada a todo tipo de tecnologías (impresas, gráficas, sonoras, pantallas y otras) (Dobudko et al., 2019a). El desarrollo de la competencia mediática se acompaña del desarrollo del pensamiento creativo, las habilidades de reflexión, la formación de la experiencia personal y le permite: analizar y comprender críticamente textos, interpretarlos y seleccionarlos para resolver problemas específicos, utilizarlos para desarrollar sus proyectos (Eliseeva et al., 2020). La competencia mediática resuelve los problemas de formación de los estudiantes en nuevas condiciones innovadoras de información, percepción operativa y procesamiento de la información. Realiza las siguientes funciones: social-adaptativa, orientada a valores, orientada a la actividad y comunicativa (Klimenko, 2012).

Entre los indicadores de competencia mediática se encuentran: motivacional (motivos para el contacto con varios medios), contacto (frecuencia de interacción con los medios), informativo (conocimiento del aparato terminológico asociado a los medios), perceptual (capacidad para percibir el texto mediático), interpretativo (capacidad para evaluar críticamente las tecnologías innovadoras utilizadas y aplicarlas en situaciones específicas), actividad (capacidad para elegir herramientas electrónicas y utilizarlas en el proceso de aprendizaje), creativa (resolver problemas profesionales de forma no estándar con el uso de tecnologías de la información) .

\section{Metodología}

La investigación tiene como objetivo estudiar la formación de la competencia mediática de los estudiantes universitarios. Para ello se seleccionaron los niveles que se presentan en la tabla.

Cuadro 1. Niveles de formación de la competencia mediática.

\begin{tabular}{cl}
\hline Nivel & \multicolumn{1}{c}{ Caracteristicas } \\
\hline Alto & $\begin{array}{l}\text { El estudiante utiliza libremente los medios de las tecnologías de la información y la } \\
\text { comunicación en el proceso educativo, está listo para aplicar la experiencia adquirida en el } \\
\text { campo profesional, participa activamente en la creación de proyectos de medios, utiliza } \\
\text { tecnologías electrónicas modernas para completar tareas, se da cuenta de su esencia y la } \\
\text { necesidad de una mayor implementación }\end{array}$ \\
\hline Medio & $\begin{array}{l}\text { El estudiante utiliza los medios de las tecnologías de la información y la comunicación en el } \\
\text { proceso educativo, está listo para aplicar la experiencia adquirida en el campo profesional, } \\
\text { participa en la creación de proyectos de medios, utiliza tecnologías electrónicas modernas } \\
\text { para completar las tareas, se da cuenta de su esencia y la necesidad. para una mayor } \\
\text { implementación }\end{array}$ \\
\hline Bajo & $\begin{array}{l}\text { El estudiante se da cuenta de la esencia de las tecnologías de la información y la } \\
\text { comunicación en el proceso educativo, pero no quiere participar en la creación de proyectos } \\
\text { de medios, utiliza tecnologías electrónicas modernas para completar las tareas. }\end{array}$ \\
\hline
\end{tabular}


La confiabilidad de los resultados de la dinámica de formación de la competencia mediática en los grupos control y experimental se determinó probando la hipótesis estadística de comparar los promedios según la prueba t de Student. Este método contribuye a la interpretación más eficaz de los resultados de la medición. La comparación de los valores de los datos obtenidos en los grupos control y experimental se calculó la prueba t de Student mediante la fórmula:

$$
t=\frac{M_{1}+M_{2}}{\sqrt{\frac{\sigma_{1}^{2}}{N_{1}}+\frac{\sigma_{2}^{2}}{N_{2}}}}
$$

$\mathrm{N} 1$ : los datos aritméticos promedio de las pruebas realizadas en el grupo de control, N2: los datos aritméticos promedio de las pruebas realizadas en el grupo experimental. $\sigma \_1 \wedge 2$ es la varianza del grupo de control, $\sigma \_2 \wedge 2$ es la varianza del grupo experimental. N1 - el número de personas en el grupo de control, $\mathrm{N} 2$ - en el grupo experimental.

El número de grados de libertad para la interpretación de la prueba t de Student se calculó mediante la fórmula:

$$
f=\left(N_{1}+N_{2}\right)-2
$$

A continuación, se determinó el valor crítico de la prueba t de Student para el nivel de significancia requerido $(p=0.05)$ y el número de grados de libertad $f$ obtenido según la tabla. Como resultado, la media aritmética del conjunto comparación de las respuestas, la varianza, el número de grados de libertad, y la prueba t de Student como se obtiene.

\section{Resultados y discusión}

La formación de la competencia mediática se lleva a cabo teniendo en cuenta las características individuales de los estudiantes, el nivel inicial de formación de la competencia mediática en el proceso de varios tipos de actividades. Cada alumno conoce el campo de las tecnologías de la información, porque durante mucho tiempo se ha introducido en todos los ámbitos de la sociedad y el alumno tiene la habilidad de utilizar herramientas electrónicas. La tarea principal de la formación en una institución de educación superior en la formación de la competencia mediática es la formación de la capacidad de utilizar tecnologías electrónicas en actividades profesionales. En el proceso de aprendizaje, los estudiantes utilizan las capacidades de "nubes", servicios de red, como Learning Apps.

En el proceso de preparar a los estudiantes para conferencias, clases prácticas y realizar trabajos independientes, se utilizan varios programas y servicios.

El estudio utilizó la prueba t de Student, que permitió determinar la confiabilidad de los resultados de la dinámica de formación de competencias mediáticas en los grupos 
control y experimental. Como resultado del análisis de los resultados del experimento, se encontró que la población general representada por las muestras de los datos obtenidos se distribuye según una ley cercana a la normal.

La tabla muestra los resultados de los cálculos.

Cuadro 2. Resultados de los cálculos sobre la formación de la competencia mediática en 2018

\begin{tabular}{lll}
\hline Grupos & Control & Experimental \\
\hline Competencia mediática & & $\mathrm{M}=32.1$ \\
\hline $\begin{array}{l}\text { La media aritmética de la población de } \\
\text { Respuestas comparadas }\end{array}$ & $\mathrm{M}=15.2$ & $\sigma=1,603$ \\
\hline Dispersion de datos individuales (varianza) & $\sigma=1,217$ & \\
\hline Número de grados de libertad & $\mathrm{f}=293$ & \\
\hline Prueba t- Student's t & 7.352 & \\
\hline
\end{tabular}

Cuadro 3. Resultados de los cálculos sobre la información de la competencia mediática en 2019

\begin{tabular}{lll}
\hline Grupos & Control & Experimental \\
\hline Competencia Mediática & & \\
\hline $\begin{array}{l}\text { La media aritmética de la población de } \\
\text { Respuestas comparadas }\end{array}$ & $\mathrm{M}=16,2$ & $\mathrm{M}=34.2$ \\
\hline Dispersion de los datos indivuales (varianza) & $\sigma=1,312$ & $\sigma=1,721$ \\
\hline Número de grados de libertad & $\mathrm{f}=295$ & \\
\hline Prueba t- Student's t & 7.361 & \\
\hline
\end{tabular}

Cuadro 4. Resultados de los cálculos sobre formación de la competencia mediática en 2020

\begin{tabular}{lll}
\hline Grupos & Control & Experimental \\
\hline Media competence & & \\
\hline $\begin{array}{l}\text { La media aritmética de la población de } \mathrm{M}=17.4 \\
\text { respuestas comparada }\end{array}$ & $\mathrm{M}=36.2$ \\
\hline Dispersión individual de datos (varianza) & $\sigma=1,412$ & $\sigma=1,721$ \\
\hline Número de grados de libertad & $\mathrm{f}=298$ & \\
\hline Prueba t- Student's t & 8,131 & \\
\hline
\end{tabular}

La dinámica de la formación de competencias mediáticas se muestra en el diagrama. 


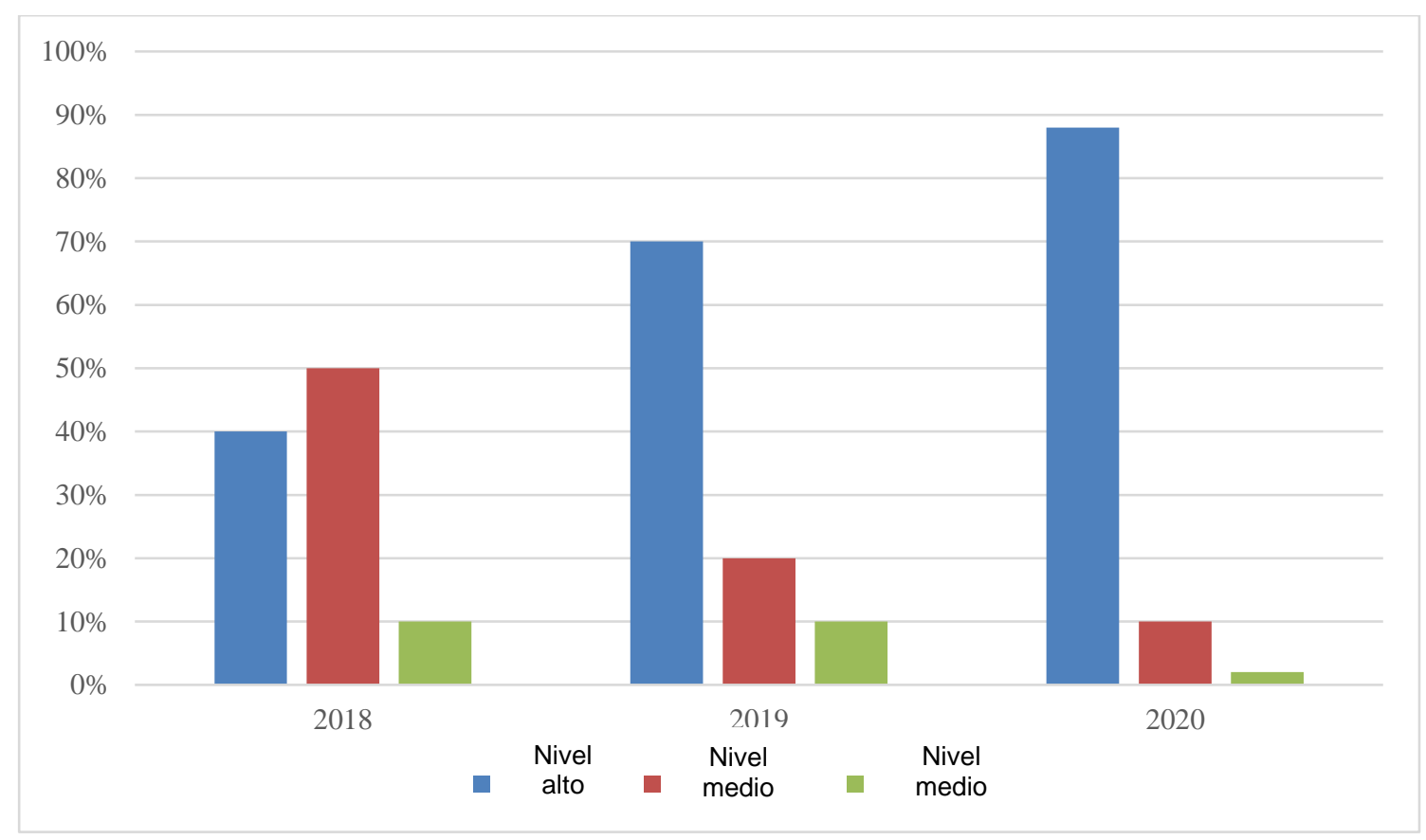

Fig. 1. Dinámica de la formación de la competencia mediática de los estudiantes en el período de 2018 a 2020

Los resultados de comprobar la formación de la competencia mediática nos permiten hablar de un aumento de su nivel para 2020. La mayoría de los estudiantes hasta el año 2020 tiene $\mathrm{n}$ un alto nivel de competencia media.

\section{Conclusion}

La mejora del entorno educativo y su saturación con tecnologías electrónicas contribuyó a un aumento en el nivel de competencia mediática de los estudiantes. En su preparación utilizaron tecnologías electrónicas modernas, plataformas educativas, aplicaciones de interacción y realización de webinars, y otros medios que permiten a los estudiantes realizar tareas de forma remota. En el proceso de aprendizaje a distancia, los estudiantes participaron activamente en el desarrollo de proyectos, concursos profesionales, conferencias de varios niveles utilizando aplicaciones para la interacción remota. La competencia mediática de los estudiantes se formó a lo largo de todo el período de estudio. Las tecnologías electrónicas son una parte integral de ella. Los estudiantes los utilizaron al realizar diversas actividades. La prueba t de Student permitió determinar la confiabilidad de los resultados de la dinámica de formación de competencias mediáticas en los grupos de control y experimentales. El valor de la prueba $\mathrm{t}$ de Student supera su valor crítico, por lo que podemos hablar de la significancia estadística de los valores sujetos a comparación y la fiabilidad de los datos obtenidos. El estudio, confirmado por datos estadísticos, muestra una tendencia positiva en la formación de competencia mediática. 
Esta investigación puede servir como base para estudiar la competencia mediática en diferentes áreas temáticas.

\section{Referencias}

Bogdanova, A.V., Korostelev, A.A., Mukhutdinov, R.H., Shakirova, I.A., \& Maseleni, A. (2019). Formulation of the problem of mathematical modeling of accommodation of basic stations of cellular communication in residential territories for students of it-directions of preparation. International Journal of Recent Technology and Engineering, 7(6), 87-90.

Dobudko, T.V., Korostelev, A.A., Gorbatov, S.V., Kurochkin, A.V., \& Akhmetov, L.G. (2019a). The organization of the university educational process in terms of digitalization of education. Humanities and Social Sciences Reviews, 7(4), 1148-1154.

Dobudko, T.V., Korostelev, A.A., Pugach, O.I., Ippolitova, N. V., Khayrullina, R.G., \& Sitdikov, F.F. (2019b). Training of pedagogical education masters: Practiceoriented model. Humanities and Social Sciences Reviews, 7(4), 1155-1159.

Eliseeva, D.Yu., Fedosov, A.Yu., Agaltsova, D.V., Mnatsakanyan, O.L., \& Kuchmezov, Kh.Kh. (2020). The evolution of artificial intelligence and the possibility of its application in cyber games. Amazonia Investiga, 9(28), 123-129. https://amazoniainvestiga.info/index.php/amazonia/article/view/1043

Grigoriev, S.G. (2005). Informatization of education. Fundamental foundations. M.: MGPU.

Kidina, L. M. (2020). Management of the pedagogical collective in the conditions of implementing distance learning. Baltic Humanitarian Journal, 9, No 4 (33), 93-96.

Klimenko, V.A. (2012). Professional socialization of students: structural and functional model. Sociological Almanac, 3, 92-102.

Konovalova, N.A. (2004). Development of media culture of students of a pedagogical university, (dissertation of the candidate of pedagogical sciences), Vologda State University, Vologda. $205 \mathrm{p}$.

Korostelev, A. A., Morozova, I. M., Gruzdeva, M. L., Smirnova, Z. V., Vaganova, O. I., Chanchina, A. V., \& Maltseva, S. M. (2019). Modern Information and Communication Technologies in the Advanced Education of Children. International Journal of Innovative Technology and Exploring Engineering, 8(9), 2376-2382.

Nagovitsyn, R.S., Vaganova, O.I., Kutepov, M.M., Martyanova. L.N., Kosenovich, O.V, Moeseev, Yu.V., Vorotova, M.S., \& Osipov, A.Y. (2020). Interactive Technologies in Developing Student's Motivation in Physical Education and Sport. International Journal of Applied Exercise Physiology, 9(6), 78-85.

Petrova, S.V. (2018). Formation of media competence of teachers of humanitarian and artistic disciplines in the system of additional education, (dissertation of the candidate of pedagogical sciences), Taganrog Institute named after AL. Chekhov (branch) FGBU VO "Rostov State Economic University (RINH)", Rostov-on-Don. 219 p. 
Pichugina, G.A; \& Bondarchuk, A.I. (2019). Structure of the training case in the organization of the educational process. Humanitarian Balkan Research, 2(4), 5-7.

Rojas Bahamón, M., Arbeláez-Campillo, D.F., \& Prieto, J.D. (2019). The investigation as an environmental education strategy. Revista De La Universidad Del Zulia, 9(25), 89-97. Recuperado a partir de https://www.produccioncientificaluz.org/index.php/rluz/article/view/29743

Shcerbakova, E.V., \& Shcerbakova, T. N. (2019). Experience of use of remote computer technologies at the organization of independent work of students in the conditions of a mark and rating system. Baltic Humanitarian Journal, 8, No 4 (29), 192-195.

Vaganova, O., Rudenko, I., Markova, S., Smirnova, Z., \& Kutepov, M. (2019). The use of educational video materials in educational process of a higher educational institution. Amazonia Investiga, 8(22), 216-222. Retrieved from https://amazoniainvestiga.info/index.php/amazonia/article/view/308

Yarygin, O.N., Korostelev, A.A, Mukhutdinov, R.H., \& Maseleno, A. (2019b). Elections and Russian citizens residing overseas: Prospects for internet voting. International Journal of Recent Technology and Engineering, 7(6), 52-57

Yarygin, O.N., Korostelev A.A., Akhmetov, L.G., \& Maseleno, A. (2019a). Modeling of competence as a tool of goal setting for education in modern society. International Journal of Recent Technology and Engineering, 7(6), 72-77.

Zmanovskaya, N.V. (2004). Formation of media education of future teachers (dissertation of the candidate of pedagogical sciences), Irkutsk State Linguistic University, Irkutsk, $152 \mathrm{p}$. 